\title{
Introduction Virtuality and Virtualization
}

\author{
Kevin Crowston ${ }^{1}$ and Sandra Sieber ${ }^{2}$ \\ 1 Syracuse University School of Information Studies \\ Syracuse, NY 13244 USA \\ crowston@syr.edu \\ 2 IESE Business School \\ Avda. Pearson 21, 08034 Barcelona, Spain \\ sieber@iese.edu
}

\section{Introduction}

In today's rapidly changing global work environment, all workers directly experience increased organizational complexity. Companies are functionally distributed, many across the globe. Intense competition for markets and margins makes adaptiveness and innovation imperative. Information and communication technologies (ICT) are pervasive and fundamental infrastructures, their use deeply integrated into work processes. Workers collaborate electronically with co-workers they may never meet face-to-face or with employees of other companies. New boundaries of time, space, business unit, culture, company partnerships, and software tools are driving the adoption of a variety of novel organizational forms. On a macrolevel, these changes have started to reshape society, leading some to speak of the "Network Society" and the "Information Age."

The word "virtual" has become a compelling catchphrase to describe these changes, but with different underlying meanings. "Virtual" is used to identify emergent work forms that differ from traditional work on dimensions such as the location of the workers, where and how work is done, how workers and teams or managers interact, and the relationships between partner organizations. "Virtual" can describe work environments where individuals are dispersed in time and space. Examples are individuals working at home (telecommuting), teams of employees from different organizations managing a supply chain or a shared project, or organizations that are established only for a certain time for a concrete purpose. Teams may disband when a project is over, and individuals may work on several teams at a time. Finally, these novel work arrangements may be called "virtual" because the work is done via ICT with simulated images and processes rather than exchanges of physical materials and performance of physical processes.

While there is broad agreement about the nature of these changes, their scope and significance demands more in-depth research and debate. The increasing reliance on computer-mediated interaction has been heralded by some as the emergence of a new organizational form, while others have criticized this perspective as techno-utopian, pointing out that in fact, organizations and individuals often resist attempts to change, or change in unanticipated ways. The phenomenon of virtuality highlights 
the dual nature of technology, in which human action and the social context in which the action takes place both shape technology, while simultaneously technology influences human actions and social structures. This perspective invites us to reflect more deeply on the nature, direction, and future of technology, organizations, individuals, and virtuality. The papers in this book contribute to this reflection, addressing a wide range of topics relating to our understanding of virtuality and virtualization, from leadership to processes to fiction.

\section{Frameworks for Understanding Virtuality and Virtualization}

The book begins with consideration of possible frameworks for understanding virtuality and virtualization. D'Eredita and Nilan examine the phenomena associated with virtual collaborative work and present a framework for understanding issues related to virtual teams and their use of information technology. They note that a team focused on some problem requires participants to engage in sense-making, sense-giving, and organizing in order to generate collaborative action. An implication of this perspective is that systems for virtual teams should explicitly support rather than take for granted these fundamental processes.

Jha and Watson-Manheim turn attention to the firm level, reviewing papers on virtual organization in terms of the type and goals of the organization and the strategies employed to manage this kind of virtual work. Although they found very little empirical research, the conceptual work suggests that the strategies employed vary with the goals of the organization: virtual organizing for abstract resources had decentralized network structure and collaborative ties with partners, while virtual organizing for specific goals had centralized network structure and opportunistic ties.

This topic is also addressed by two panels, which are described briefly in the final section of the book. In the first, "Virtualization and Institutions," panelists Barrett, Davidson, Silva, and Walsham explore how institutional theory might be valuable for understanding virtualization of work practices. In the second, "Exploring the Nature of Virtuality," panelist Panteli, Chiasson, Yan, Poulymenakou, and Papargyris examine the multi-dimensional nature of what virtuality has been, is, and may become, and specifically its global and local dimensions, as well as the different interpretations that are given to these dimensions.

\section{Process Issues to Achieve Virtualization}

The book next includes papers that consider ways of analyzing virtual work in terms of work processes. Nilan and Mundkur describe a procedure for generating user-based cognitive and social cognitive models of tasks/problems/contexts that can be employed to create readily navigable link structures for virtuality-mediated communication and collaboration purposes. Based on the description by 128 respondents of the steps taken during an interactive e-Commerce situation, they develop a model of e-Commerce as a series of logically necessary steps over time. 
Katzy and Crowston describe the organizational activities necessary for firms to cooperate within a virtual organization called the Virtuelle Fabrik (the Virtual Factory), which reliably engineers and delivers manufacturing projects. Firms in the network can access market opportunities and additional needed competencies through a process they call "competence rallying." They argue that the success of manufacturing projects in this virtual organization is predicated on specific organizational activities in four phases of the competence rallying process: 1) identification and development of competencies, 2) identification and facing of market opportunities, 3) marshalling of competencies, and 4) a short-term cooperative effort.

Consideration of work processes leads to consideration of the coordination of those processes. Cummings, Espinosa, and Pickering note that spatial (distance) and temporal (time zones) boundaries affect collaborative work by making informal and synchronous communications more difficult, respectively. They use social network data from 615 team members (representing 5919 pairs) across 137 global teams in a multi-national semiconductor firm to explore the impact of these boundaries on coordination delay and team outcomes. They found, as expected, that boundaries increased delays and decreased outcomes, and while communication helped reduce these effects, it did not help distributed pairs more than others.

Three papers examine coordination issues through in-depth case studies. First, Wiredu examines how global software development units deal with uncertainty from other units. From a case study of a software developer, he suggests that variety in information systems is needed for managing these uncertainties. Specifically, he notes five characteristics of variety that are needed: agility of developers, continuity of developers, high frequency of communications, varied communication modes and technologies, and virtuality that makes global expertise accessible.

Ocker, Huang, Trauth, and Purao discuss the complexities of accomplishing knowledge work within a hybrid team configuration in which members alternate between co-located and distributed contexts, with differing levels of availability. From a case study of a research team writing a paper together, they identify reasons for member unavailability and contrast them with the expectation of availability.

Finally, Sharma and Krishna investigate the level of process maturity in geographically dispersed software sustenance activities. They report on a case study of one organization that maintains proprietary software from three different locations. From this case, they describe a variety of processes that were adopted to support the organization, including carefully documented processes supported by shared workspaces.

\section{Group Processes in Virtual Teams}

The third section includes papers that examine group processes within virtual teams, focusing in particular on leadership and group identity. Heckman, Crowston and Misiolek present a two-order theory of leadership in virtual teams, building on behavioral leadership theory and structuration theory. Their theory describes four classes of first-order leadership behaviors (task coordination, substantive task contribution, group maintenance, and boundary spanning), and defines second-order 
leadership as behavior that affects changes in the structures that guides group action. They propose that effective virtual teams will exhibit a paradoxical combination of shared, distributed first-order leadership complemented by strong, concentrated, and centralized second-order leadership.

Reilly and Ryan introduce the notion of Ambassadorial Leadership, arguing that leaders of globally distributed virtual teams should have the characteristics of an Ambassador, someone who is culturally sensitive, able to span boundaries created by geography and functional background, and able to help build a collective identity for the virtual team. They develop an instrument for assessing Ambassadorial Leadership and test its measurement properties in a small pilot study.

Finally, a set of papers examines how virtuality affects individuals' identity and involvement in their work. Vaast applies Goffman's theoretical perspective to investigate how people present themselves in the virtual yet work-related environments of occupational online forums. Specifically, she analyzes the profiles of more than 300 registered users of an online forum dedicated to issues of interest to bankers. Four categories of profiles emerged, which she names after the typical characters in a play: Protagonist (the leading character), Deuteragonist (a secondary character), Tritagonist (a minor character whose specific background the audience is not made aware of), and Fool (a humorous character).

Brooks also argues that Goffman's terminology and concepts afford a powerful way of integrating the study of virtual action and interaction with the study of social action and interaction more generally. Specifically, she considers how participants in virtual environments interact with each other, as they would in co-present situations, and argues that several important aspects of virtuality can be well-accounted-for by this approach: the non-virtual "Real World," the meaning of simulated images and processes, immersion in simulated images, and processes and virtual social interaction.

Williams, O'Leary, and Mortensen note that increasingly people are members of more than one team at a time and they, their team leaders, and organizations must manage the challenges posed by relying on multiple team memberships (MTMs) as a way to structure work. From interviews and a survey of 401 professionals about the prevalence and nature of MTM in their work, they find that MTM is quite common, and requires individual members and team leaders to negotiate competing demands.

The conference also includes a panel organized by Chong, Erickson, Lee, and Siino. "The Social in the Virtual" discusses social elements of virtual environments, such as how social presence, influence, and awareness operate in virtual environments and the interplay between social patterns and structures in the physical and the virtual.

\section{Knowledge and Virtuality}

In the fourth section of this book, we consider the role of knowledge in virtual settings, considering its impact on group learning and competence management issues, as well as the impact of cultural differences. Annabi argues for the importance of group learning for virtual group effectiveness. To examine how and when virtual groups learn, she studies the developers of the Apache Web Server, 
who are geographically and organizational distributed. From the email transcripts of the group, she develops a taxonomy of what she calls learning triggers, events that initiate learning opportunity episodes by demonstrating the possibility for the group to learn to perform better, for example, a problem or the realization of a possible innovation.

Wei uses a case study to explore Chinese workers' perceptions of the impact of national cultural difference (China vs. U.S.) on knowledge sharing activities in global virtual teams in a high-tech company. Four cultural dimensions (language, education, technology and material culture, and attitudes and values) are identified. Her results show that language has the most salient impact on individuals' knowledge sharing activities, followed by education, attitudes and values, and technology and material culture. Individual characteristics, organizational culture, time zone problem, and leadership style have a mediated impact on the knowledge sharing activities.

Scott and Venters consider the implications of virtuality for our own knowledge development practice, by examining developments in e-science (that is, virtualization of research practice) and in e-social science in particular. As they state, "The standards and shared approaches implicit in engaging with e-social science enable the exciting prospect of conducting large scale research in ways not possible before." However, they caution that such innovations also create the need for novel kinds of research efforts (e.g., data curation), require a rethinking of the standards and norms for our own work (e.g., increasing the need for research teams rather than solo investigators), and potential privilege of certain kinds of work (e.g., that based on easily-shared quantitative data).

Westergren examines strategies that an organization can use to keep competences within a client organization when outsourcing services. She examines this question in a study of the relationship between a large minerals company and its service provider. She documents several strategies employed to maintain competences, such as exploiting the full potential of the partnership in order to learn from the supplier, and heavy investment in information technology.

Oshri, Kotlarsky, van Fenema, and Willcocks explore management of expertise in offshore outsourcing projects and conclude that it consists of three key processes, namely development, coordination, and integration. They find evidence for these processes in a case study of the ABN AMRO bank outsourcing to TATA Consultancy Services. They suggest that the management of expertise in such projects involves the coordination and integration of expertise that is both locally and globally developed, within and across projects.

A panel organized by Majchrzak and Wagner brings together professionals in both industry and academia to discuss "The Role of Shapers in Knowledge Sharing" The panelist define shaping (or gardening) as involving dynamically editing, integrating, distilling, refactoring, identifying areas of convergence and discrepancies, identifying topics receiving little attention in the community, and significantly rewriting the contributions of others. 


\section{The Role of Fiction in Structuring Virtuality}

Virtuality can imply simulated images and processes, and a final set of papers considers the role of such fiction in structuring virtuality. Tapia, El-Nasr, Zupko, and Maldonado evaluate the efficacy of building virtual environments for influencing girls' interest in computer-related careers. Specifically, they developed a set of courses for middle and high school girls they in which they taught technology skills including programming, design and visual editing through developing video games. They find that the use of virtual environments and games captures the attention of participants, increase self-efficacy, and inspire some to consider computing as a career.

Rennecker and Schultze reconceptualize synthetic worlds such as Second Life as an emergent communication medium. They differentiate synthetic worlds on two dimensions-realism vs. fantasy and progression vs. emergence structure-- to differentiate four kinds of worlds, but argue that all are a legitimate form of communications medium. They suggest possible research questions that IS researchers could address in these environments.

Finally, Ramiller considers processes by which organizations create and enter the new world of the virtual. He notes the particular importance of fictionalizations in the constitution of the future, suggesting that they can be generative, pulling the world towards a desired imagined state. As he says, "a real organizational innovation such as virtual work reflects, for a considerable period of time, an uneasy and dynamic mix of discourse and material activity." In other words, to understand the nature of virtual work, we need to understand what he labels the narrative-virtual that organizational actors create to predict and create this future state.

A panel on "Game Architectures and Virtual Teamwork," organized by Esther Baldwin of Intel Corporation, discusses the possibilities for virtuality of "serious games," noting that game architectures offer qualities that are missing in many collaboration tools, such as multi-tasking through objects, multi-teaming through context switching and "rooms," stimulating visuals and action environments.

\section{Conclusion}

In addition to the papers and panels discussed above, the conference program includes two keynote speakers. Michael Cohen's presentation, "Beyond Distributed Cognition: Widening Our Conceptual Foundations to Better Support Virtual Organization," argues for consideration of habit and emotion (in addition to cognition) as a basis for understanding virtual organizing. John Leslie King addresses "Dig the Dirt: Hashing Over Hygiene In the Artifice of the Real" to show how the "the patently 'unreal' notion of virtuality makes a rhetorical play for the status of "real" "making virtual reality just a particular kind of reality. Two additional panels discuss specific types and applications of virtuality. Panelist Bélanger, Watson-Manheim, Harrington, Johnson, and Neufeld discuss, "The IT Artifact and Telecommuting," considers how research on telecommuting addresses the information technology artifact, which has been argued to be central to information systems research. Panalists Kaplan, Elkin, Gorman, Knoppel, Sites, and Talmon 
discuss "Virtual Patients" and explore virtuality in health care environments, with a particular focus on the virtual patient. Panelists explore different aspects of how developments leading towards virtual patients point towards significant issues of virtuality in other environments. The conference closes with a summative conversation between General Chair Wanda Orlikowski and Geoff Walsham.

Taken together, the papers and panels in this book present an impressive snapshot of the current state of the art in research on virtuality and virtualization.

\section{Acknowledgements}

This conference is itself has been the product of a virtual organization that has benefited from many distributed and voluntary contributions. We thank all those who helped shape the conference: the IFIP Working Group 8.2 and 9.5 executives and members who approved the conference, all those who submitted papers and panel proposals, the program committee and additional reviewers, our organizing chair, Eleanor Wynn and our general chair, Wanda Orlikowski. Sherri Roberts (sherritr@yahoo.com) ably edited these proceedings. We also acknowledge the support of our sponsors, Cynthia Pickering and Bert Cave of Intel Corporation, and Portland State University, without whom the conference would not be what it is. This conference, the result of these many distributed contributions, exemplifies the powerful possibilities of virtual work. 